\title{
PROTON AND LIGHT ION INTERACTIONS IN COSMIC RAY EXPERIMENT "STRATOSPHERE "IN THE COMPARISON WITH THE RECENT COLLIDER RESULTS
}

A.Kh. Argynova ${ }^{a}$, N.I. Kochelev ${ }^{b}$, T.N. Kvochkina ${ }^{c}$, A.A. Loktionov ${ }^{a}$,T.Kh. Sadykov ${ }^{a}$, N.N. Zastrozhnova ${ }^{a}$, Y.M. Tautayev ${ }^{* a}$, S.Zh. Tokmoldin ${ }^{a}$

${ }^{a}$ Institute of Physics and Technology

Almaty, Kazakhstan

${ }^{b}$ Joint Institute for Nuclear Research

Dubna, Russia

${ }^{c}$ Institute of Nuclear Physics

Almaty, Kazakhstan

E-mail: kochelevegmail.com, loctionesci.kz, turlan43@mail.ru

The interactions of cosmic ray light nuclei and protons with different targets were studied at "Stratosphere" experiment at energies above $10 \mathrm{TeV}$ in the Lab system. The results show that in the rare events, produced by alpha-particles and light nuclei, transverse momentum spectra of secondary $\gamma$-quanta in the soft region (up to $2 \mathrm{GeV} / \mathrm{c}$ ) have exponential character with large values of inverse slope of the distributions: $T_{A} \sim 0,8 \mathrm{GeV} / \mathrm{c}$. On the contrary, in the proton interactions the slope is essentially smaller $T_{p} \sim 0,2 \mathrm{GeV} / \mathrm{c}$. For charged secondary particles the high order intermittency analyses have again demonstrated the large difference between events produced by protons and nuclei. So, essential system size dependence in the forward production dynamics was obtained with the limited statistics.

Such processes with a large energy flux in the forward direction are difficult to measure at the Collider experiments. Current Large Hadron Collider forward (LHCf) experiment and future RHICf experiment are very important to give the information about the dynamics of strong interaction in both , high energy heavy ion physics and in high energy cosmic rays. However, there are different serious problems to compare directly the results of Stratosphere and LHCf experiments, including problem with different kinematics and the requirement for the study of the light nuclei interactions at the Colliders as well.

In the present paper, as the first step, we compare the data of Stratosphere and LHCf experiments for PT spectra of $\gamma$-quanta and neutral pions production in the proton-induced interactions in commensurable rapidity intervals.

The 34th International Cosmic Ray Conference,

30 July- 6 August, 2015

The Hague, The Netherlands

\footnotetext{
* Speaker.
} 


\section{INTRODUCTION}

The understanding of the processes of the particle production in hadronic interactions at high energies have a great significance, both theoretical and experimental. Theoretical models have to provide the prediction for hadroproduction in order to get a clear insight on the processes within the hot and dense strongly-interacting matter. Experimental analyses have to perform knowledge discovery for the physical properties of the excited fireball from the complex final pattern of produced particles in nucleus-nucleus collisions. The effective way to the interpretation of the results consists in the analyses of the smaller system interactions, created in proton-proton or in proton-nucleus collisions. On the basis of such approach the interactions of cosmic ray light nuclei and protons with different targets have been studied in the experiment "Stratosphere" at energies above $10 \mathrm{TeV}$ in the Lab system [1]. The results have shown that in the rare events, produced by alpha-particles and light nuclei, transverse momentum spectra of secondary $\gamma$-quanta in the soft region (up to $2 \mathrm{GeV} / \mathrm{c}$ ) have exponential character with large values of inverse slope of the distributions: $T_{A} \sim 0,8 \mathrm{GeV} / \mathrm{c}$. On the contrary, in the proton interactions the slope is essentially smaller $T_{p} \sim 0,2 \mathrm{GeV} / \mathrm{c}$. For charged secondary particles the high order intermittency analyses have again demonstrated the large difference between events produced by protons and nuclei. So, the essential system size dependence in the forward production dynamics has been obtained on the limited statistics. Similar events were observed by JASSE [2] and Concorde [3] cosmic ray collaborations.

The comparison of our cosmic ray data with a current LHCf [4-5] and with a future RHICf data [7] are very important both for frontier problems of high energy heavy ion physics and for actual high energy cosmic ray problems. However, there are some problems in the comparison of the results of Stratosphere and LHCf experiments. In the first case in the interactions proton and light nuclei with light and heavy targets PT the spectra of $\gamma$-quanta and angular distributions for charged particles have been obtained. In the second case the inclusive PT spectra for the neutral pion in the forward rapidity were studied. These experiments were performed in the slightly different intervals on energies and with exclusive and inclusive approaches to the data taking. Therefore, the detailed theoretical or Monte Carlo forward production data are need for the complete solution of these comparison problems. Furthermore, a new collider experiments are needed to perform to study the high energy interaction between light nuclei . So, in the present paper, as the first step, the only limited task will be done. We will compare spectra of $\gamma$-quanta and neutral pions in the proton-induced interactions at commensurable rapidity quantities.

We will also discuss the non-perturbative QCD mechanism which might be responsible for the difference in particle production in central and forward rapidity regions [8].

\section{EXPERIMENT "STRATOSPHERE"}

\subsection{PT spectra of $\gamma$-quanta from experiment "STRATOSPHERE"}

Cosmic ray interactions at $E_{0}>5 * 10^{13} \mathrm{eV}$ were found by Stratosphere Collaboration in X-ray film - emulsion chambers exposed in the stratosphere at altitude $\sim 30 \mathrm{~km}$. The each chamber included the target and gamma blocks. The target block was gathered of many layers of heavy or light material interplead with thin nuclear emulsions. The gamma block consisted of 4 - 10 lead plates each $5 \mathrm{~mm}$ thickness with nuclear emulsions and X-ray films between them. For each interaction 


\section{O - event generated by protons - event generated by nuclei}
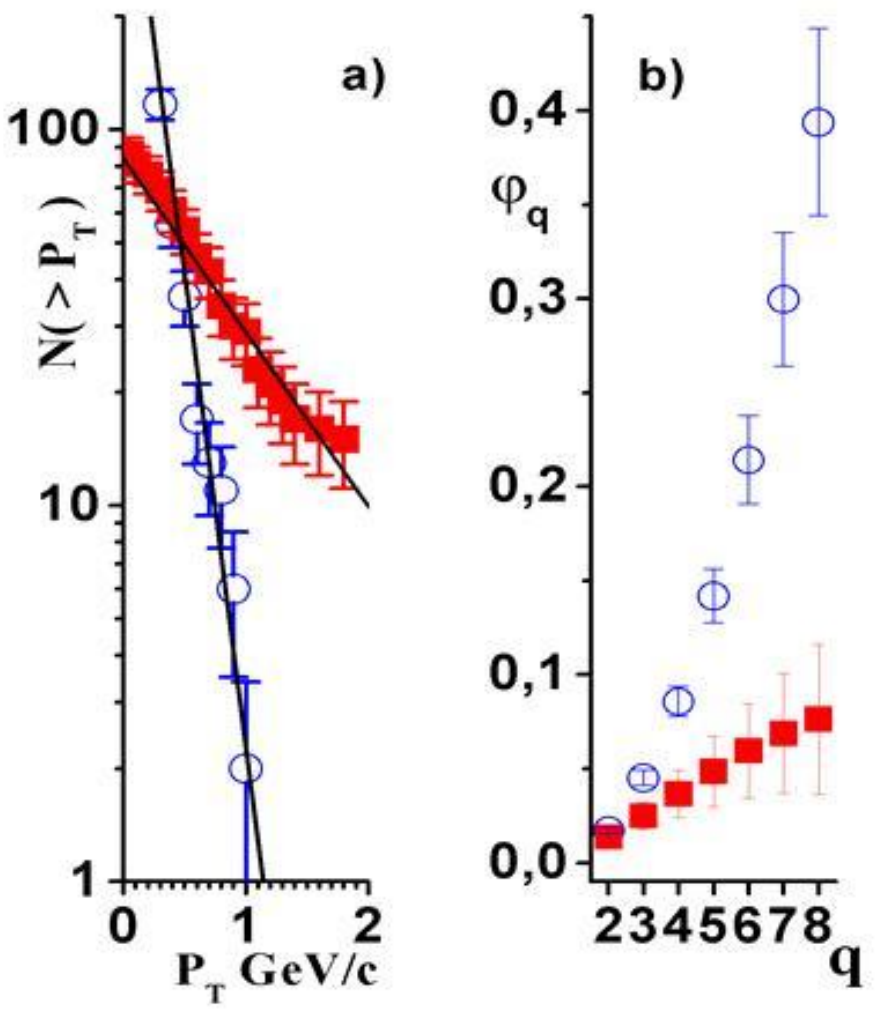

Figure 1: The integral distribution of transverse momentum of and the intermittency exponent $\varphi_{q}(q)$ be-haviour for events produced by protons and nuclei.

in the target block the number of charged secondary particles $(n c h)$, the number of secondary $\gamma$-quanta $(n \gamma)$, the angles of emission of each secondary particle in the laboratory coordinate system $\theta$ ch and $\theta \gamma$, an energy and a transverse momentum of each over threshold $\gamma$-quantum $E \gamma$ and $p t \gamma$ have been determined. The threshold for the electromagnetic cascade in X-ray films was $2 \mathrm{TeV}$ and in nuclear emulsion $-0.05 \mathrm{TeV}$. The deviation of the accuracy for $p t \gamma$ has not been greater than $30 \%$.

It can be describe by the superposition of two exponents:

$$
N_{\gamma}\left(>p_{t \gamma}\right)=A_{1} \exp \left(-p_{t \gamma} / p_{01}\right)+A_{2} \exp \left(-p_{t \gamma} / p_{02}\right)
$$

where $p_{01} \sim 0,2 \mathrm{GeV} / c$ for "standard" events and $p_{02} \sim 0,8 \mathrm{GeV} / \mathrm{c}$ for "anomalous" ones. The main feature of these "anomalous" interactions is that most of photons have the transverse momen-tum $p_{t \gamma} \geq$ $0,5 \mathrm{GeV} / \mathrm{c}$.

\subsection{Analysis of the structure of fluctuations from experiment "STR ATOSPHERE"}

The intermittency analysis of Stratosphere Collaboration data (Dobrotin et al., 1979; Apanasenko 
et al., 1990) has been performed [1]. To study dynamical density fluctuations over the background of the statistical fluctuations, the analysis of the scaled factorial moments of the multiplicity distribution (Bialas and Peschanski, 1986, 1988) has been used:

$$
<F_{q}>=\frac{<n^{[q]}>}{<n>q}
$$

where $<n^{[q]}>=<n_{m}\left(n_{m}-1\right) \ldots\left(n_{m}-q+1\right)>$ for ever decreasing domains of phase-space $\delta$, down to the experimental resolution.

If self-similar fluctuations of many different sizes exist, then the dependence of the moment $<F_{q}>$ from the size of the phase-space bin follows the power law and process is called "intermittency":

$$
<F_{q}>=1 / \delta^{\varphi_{q}}
$$

for $\delta \longrightarrow 0$. Positive constant $\varphi_{q} \neq 0$ is the so called intermittent exponent.

The distribution (2.3) is discontinues, i.e. contains sharp spikes and holes between particles in phase-space. The observation of such a power law in a sufficiently large range of scales $\delta$ would be indicate at the selfsimilar fractal structure of the short range particle density fluctuations.

In the opposite case of the smooth distribution (probability density is continuous), the factorial moments are

$$
<F_{q}>\sim \text { Const }
$$

It should be mentioned that the high order moments resolve the large tail of the multiplicity distribution. Thus, they are very sensitive to density fluctuations at the various scales $\delta$ used in the analysis. Therefore, the study of fluctuations in the large of scales $\delta$, especially at small $\delta$ and large $q$, can improve our phenomenological understanding of multiparticle production processes.

On the experiment the particle multiplicity distribution are studied for a sequence of phase space domains $\delta$ by consecutive subdivision of an initial region $\Delta$ in $M$ equal subdomains: $\delta=$ $\Delta / M$.

In order to improve the statistical accuracy in the experimental estimation of factorial moments $F_{q}^{\prime}$ s of individual cells (2.2) are averaged over events and over M cells ("vertical analysis"). In our work we used the modified method of vertically averaging (Argynova et al., 1997) in which moments are averaged over the start point of the original region $\Delta$ location

$$
<F_{q}>=\frac{1}{M} \sum_{m} \frac{1}{N_{\text {step }}} \sum_{\text {step }} \frac{1}{N_{\text {evt }}} \sum_{\text {evt }} \frac{<n_{m}^{[q]}>}{\left.<n_{m}\right\rangle^{q}}
$$

where $N_{\text {step }}$ is the number of small $($ step $/ \Delta \ll 1)$ steps of the start position of the original region $\Delta$ in the area of pionization. In every subdomain $m(m=1, \ldots, M) n_{m}$ is the multiplicity of that bin. As the basic variable the pseudo-rapidity $\eta=-\ln \operatorname{tg} \theta / 2$ of the charge secondary particles has been used. The initial region of $\Delta$ was 4.0 and $M=40$.

From our point of view the lowest order of correlations are not very sensitive to the recognition of instanton-induced processes and only the consideration of high orders can give the correct 

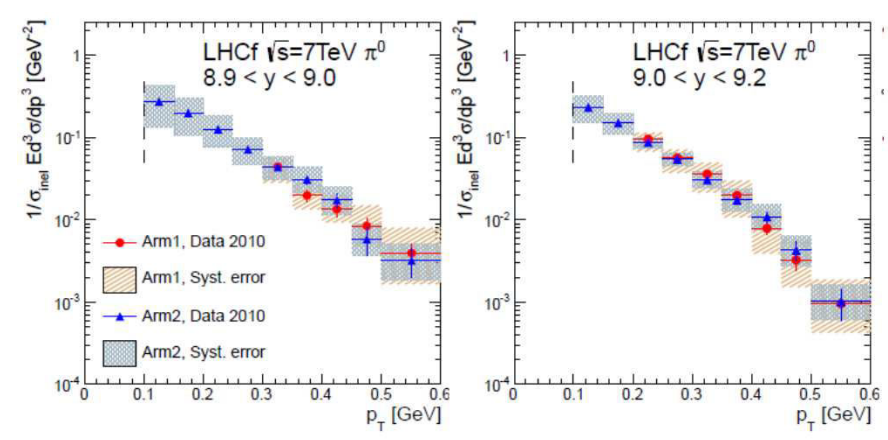

Figure 2: LHCf 2010 data, proton-proton collisions, Forward neutral pion transverse momentum spectra with rapidity range greater than $y=8.9$

results. Therefore, the high order intermittency analysis with rank of moments up to 8 has been performed. For events with standard $p_{t}$ distribution the results of the analysis has shown that the $\ln F_{q}^{\prime}$ s rise with increasing $-\ln \delta \eta$ for all orders. The data are consistent with intermittent behaviour, i.e. with power law (2.3). On the contrary, for large $p_{t}$ events the values of the slopes and their changing as function of $q$ are essentially smaller. The results of the fit of the $\ln F_{q}^{\prime}$ s as a function of $-\ln \delta \eta$ (over $0.1 \leq \delta \eta \leq 1.0$ ) have shown that the all slopes $\varphi_{q}$ are essentially larger for the events with small $p_{t}$ in comparison with the case of the large $p_{t}$ events.

\section{PT SPECTRA OF FORWARD NEUTRAL PION FROM LHCf EXPERIMENT}

The launch of the Large Hadron Collider (LHC) opens a new possibilities for high energy physics at the $\mathrm{TeV}$ scale. The previous RHIC explorations on the soft physics at midrapidity [10] were developed in the work of the ALICE collaboration [11].

In the very forward region the new experiments were performed at LHC forward detector LHCf. In proton-proton collisions at $900 \mathrm{GeV}$ and at $7 \mathrm{TeV}$ transverse momentum distribution for inclusive neutral pions has been measured [4] and in p-Pb collisions at $5.02 \mathrm{TeV}$ [5].

All proton induced data (with antiproton-proton collisions at $630 \mathrm{GeV}$ from UA7 experiment) show that there is the weak dependence of average value of the neutral pion PT distribution up to LHC energy range [6]. With this result in mind it should be concluded that exponential fits for the spectra $[4,5]$ well enough coincide with the correspond estimation from our Stratosphere experiment, given upper bound.

In the proposal [7] a new forward particle production experiment PHENIX-RHICf was suggested, in which $p-p$, proton-Nitrogen, and Nitrogen-Nitrogen, Fe-Nitrogen reactions, as a future options, were considered. These experiments at RHICf energies will provide a lower bound for the Stratosphere experiment estimations.

\section{NON-PERTURBATIVE MECHANISM FOR THE PARTICLE PRODUCTION IN STRONG INTERACTIONS}

Recently a new mechanism for the pion production in high energy reactions with hadrons was 

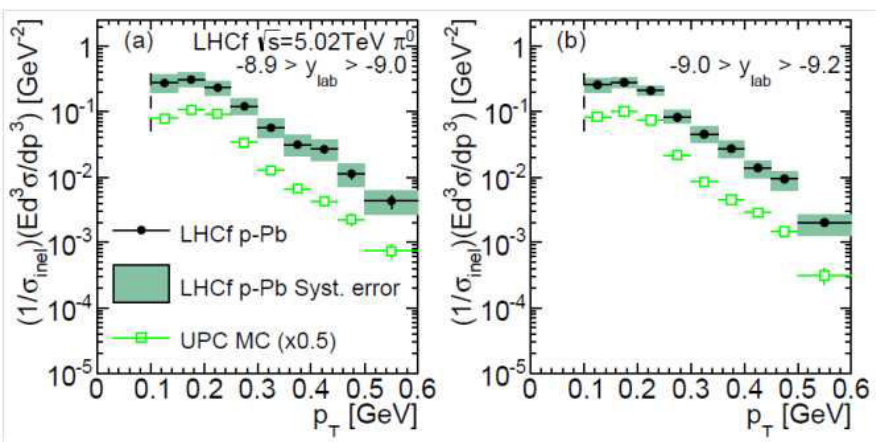

Figure 3: LHCf January 2013 data, proton-lead collisions, Forward neutral pion transverse momentum spectra with rapidity range greater than $y=8.9$

suggested [8].

This mechanism is based on the instanton model for the QCD vacuum [9].

The instantons describe the subbarrier transitions between the classical QCD vacua with the different topological charge. The existence of instantons is very important for hadron physics. For example, they provide a natural mechanism of the spontaneous chiral symmetry breaking (SCSB) in the strong interaction. As the result, large dynamical quark masses arise. One of the places where the SCSB effects might be important is the high energy reactions with hadrons. In particular, it was demonstrated that in the instanton effects give a significant contribution to the inclusive pion production in forward direction in the high-energy proton-proton scattering [8]. These effects come from the anomalous the chromomagnetic quark-gluon interaction induced by instantons [12]. The average PT for the secondary particles in the instanton induced interaction is expected to be larger in the comparison with the average PT for usual soft interaction which is related to the confinement dynamics. It comes from smaller size of instantons $(0.3 \mathrm{fm})$ in QCD vacuum to to respect to the confinement scale $(1 \mathrm{fm})$.

Therefore, the two different slopes in PT distribution for the secondary particles which were observed by the "STRATOSPHERE", might be related to those different mechanisms.

\section{SUMMARY}

The comparison of the PT spectra of the neutral particles production in the proton-induced interactions of the Stratosphere and LHCf experiments shows that there is some agreement between their slopes (exponential fit) for corresponding rapidity intervals. LHCf data in this case can be considered as an upper bound. We discuss also the possible non-perturbative QCD mechanism for the particle production in forward direction in high energy reaction with hadrons and nuclei. 


\section{References}

[1] A. Kh Argynova et al., Proc. of 27-th ICRC, p.1477-1480, Hamburg, 2001.

[2] The JACCE collaboration, Phys.Rev.Lett. v.57, N 26, 1986.

[3] The Concorde collaboration, J.Phys. G.12, p.125, 1986; J.Phys.G.14, p.503, 1988

[4] O. Adriani et al., - LHCf collaboration, arXiv: 1205.4578.

[5] O. Adriani et al., - LHCf collaboration, arXiv: 1403.7845.

[6] 6. M. Hiroaki - for LHCf collaboration, ICRC 2013, Rio de Janeiro.

[7] Y. Itow et al., - proposal - forward particle production at RHIC, arXiv: 1401.1004, 1409.4860.

[8] N.Kochelev, H.J.Lee, B.Zhang and P.Zhang, "Anomalous pion production induced by nontrivial topological structure of QCD vacuum", arXiv:1503.05683 [hep-ph].

[9] T. Schafer and E.V. Shuryak, "Instantons in QCD", Rev. Mod. Phys. v.70 (1998) 1323.

[10] S. Esumi - Soft physics at Phenix, Progr Theor Exp Phys, 2015, 03 A104.

[11] B. Abelev et al., ALICE collaboration, pp and Pb-Pb, HAL arXiv: hal-01104892, 19 Jan, 2015.

[12] N.I.Kochelev, "Anomalous quark chromomagnetic moment induced by instantons", Phys. Lett.B 426, 149 (1998). 